\title{
Back pain, mental health and substance use are associated in adolescents
}

\section{S.J. Kamper ${ }^{1,2}$, Z.A. Michaleff ${ }^{3}$, P. Campbell ${ }^{3,4}$, K.M. Dunn ${ }^{3}$, T.P. Yamato ${ }^{1,2}$, R.K. Hodder ${ }^{1,5,6}$, J. Wiggers ${ }^{5,6}$, C.M. Williams $s^{1,5,6}$}

\author{
${ }^{1}$ Centre for Pain, Health and Lifestyle, New South Wales, Australia \\ ${ }^{2}$ School of Public Health, University of Sydney, Sydney, Australia \\ ${ }^{3}$ Arthritis Research UK Primary Care Centre, Institute for Primary Care and Health Sciences, Keele University, Keele, Staffordshire, UK \\ ${ }^{4}$ South Staffordshire and Shropshire NHS Foundation Trust, St George's Hospital, Stafford, Staffordshire, UK \\ ${ }^{5}$ Hunter New England Population Health, Hunter New England Local Health District, Wallsend, Australia \\ ${ }^{6}$ School of Medicine and Public Health, Hunter Medical Research Institute, University of Newcastle, Australia \\ Address correspondence to Steven J. Kamper, E-mail: steven.kamper@sydney.edu.au
}

\begin{abstract}
Background During adolescence, prevalence of pain and health risk factors such as smoking, alcohol use and poor mental health all rise sharply. The aim of this study was to describe the relationship between back pain and health risk factors in adolescents.

Methods Cross-sectional data from the Healthy Schools Healthy Futures study, and the Australian Child Wellbeing Project was used, mean age: 14-15 years. Children were stratified according to back pain frequency. Within each strata, the proportion of children that reported drinking alcohol or smoking or that experienced feelings of anxiety or depression was reported. Test-for-trend analyses assessed whether increasing frequency of pain was associated with health risk factors.

Results Data was collected from 2500 and 3900 children. Larger proportions of children smoked or drank alcohol within each strata of increasing pain frequency. The trend with anxiety and depression was less clear, although there was a marked difference between the children that reported no pain, and pain more frequently.

Conclusion Two large, independent samples show adolescents that experience back pain more frequently are also more likely to smoke, drink alcohol and report feelings of anxiety and depression. Pain appears to be part of the picture of general health risk in adolescents.
\end{abstract}

Keywords behaviour, children, musculoskeletal disorders

\section{Background}

Substance use and psychological distress are causes of concern in adolescents. Population-based surveys report substantial prevalence of alcohol, tobacco and illicit substance use in teenagers in numerous countries including the USA, ${ }^{1}$ the $\mathrm{UK}^{2,3}$ and Australia. ${ }^{4}$ This is of concern because the developing brain may be acutely susceptible to negative influences of toxic substances, and use in early adolescence may increase the risk of substance use disorders and mental health problems in later life. ${ }^{5,6}$ Psychological distress, including anxiety, stress and depression are major contributors to the burden of disease among adolescents. Major depression, anxiety, and conduct disorders rank third, fourth and seventh of all conditions for years lived with disability in 15-19 years old. ${ }^{7}$ Similar to substance use, these conditions are not only responsible for a concurrent burden, but are drivers of poor health into adulthood. ${ }^{8}$ It is accepted that substance use issues and mental health are closely related, although causal associations are undoubtedly complex. ${ }^{\text {? }}$
S.J. Kamper, Associate Professor
Z.A. Michaleff, Research Fellow
P. Campbell, Research Fellow
K.M. Dunn, Professor
T.P. Yamato, Research Fellow
R.K. Hodder, Research Fellow
J. Wiggers, Professor
C.M. Williams, Senior Research Fellow 
During adolescence, the prevalence of musculoskeletal (MSK) pain (pain arising from the bones, joints or muscles) in general, and back pain in particular rises steeply. ${ }^{10,11}$ Although often dismissed as trivial and transient, there is mounting evidence that adolescent back pain is prevalent, ${ }^{12,13}$ associated with a large disability burden in its own right, ${ }^{7}$ and responsible for substantial health care utilization, ${ }^{14}$ medication usage, ${ }^{15}$ school absence, ${ }^{16}$ and interference with physical and day-to-day activities. ${ }^{11}$ Of further concern is the fact that persistent episodes of pain during adolescence appear to be related to development of chronic pain in adulthood. ${ }^{17-19}$

Previous studies have reported associations between MSK pain, substance use, and poor mental health, but the relationships between them are unclear. Evidence from a systematic review shows a small but robust relationship between smoking and chronic back pain among teenagers. ${ }^{20}$ Studies investigating the relationship between pain in adolescents and alcohol consumption report inconsistent results including positive associations, ${ }^{21,22}$ no association, ${ }^{23}$ and a study that reported adolescents with pain to be less likely to drink alcohol. $^{24}$ There is robust evidence that demonstrates links between MSK pain and indicators of poor mental health, ${ }^{25}$ for example; between recurrent MSK pain and anxiety, ${ }^{26}$ MSK pain and internalizing (anxiety, depression, withdrawal) and externalizing (aggression, rule-breaking) behaviours, ${ }^{27}$ as well as evidence of mental health factors associated with, and influencing substance use. Greater clarity is required on the relationships between MSK pain, substance use, and poor mental health, specifically regarding pain type, e.g. transient pain compared to pain that is persistent.

Much of the epidemiological research into MSK pain in children does not distinguish between the presence of 'any' pain, and pain that persists or is experienced frequently. This may contribute to inconsistency in the body of literature. Because pain is a part of everyday life, for children as with adults, it is important to distinguish between pain of consequence (e.g. persistent pain, frequent pain, or pain associated with activity limitation, school absenteeism, health care seeking) and trivial, transient episodes. Stratifying pain by frequency offers one way of attempting to make this distinction.

The aim of this study was to determine whether adolescents that experience back pain more frequently were also more likely to report other indicators of adverse health risk, e.g. alcohol use, smoking, school absenteeism and indicators of poor mental health, e.g. depression and anxiety.

\section{Methods}

We used data from two samples of adolescents to address the study aim. We chose to examine the study aim independently in the two datasets as a check on generalizability of the findings.

The Healthy Schools Health Futures study (HSHF) is a cluster randomized controlled trial testing the effectiveness of a resilience intervention on substance use in high school children. The HSHF trial included 32 high schools in the Hunter New England region of NSW, Australia. Schools were eligible if they were: (i) located in disadvantaged Local Government Areas (score of $<1000$ on the SEIFA Index of Socio-Economic Advantage/Disadvantage) and (ii) coeducational with at least 400 students in Years 7-10. Comprehensive details of the study methods have been reported elsewhere. ${ }^{28}$ At follow up for the HSHF trial in 2015, Year 9 students provided data for the current study. Based on cultural advice processes within the project governance, including consultation with a cultural advice group, students identifying as Aboriginal and/or Torres Strait Islander were not asked to provide information on pain outcomes. This was because Aboriginal students were already receiving additional items relating to community and culture, and further addition to the survey was not reasonable. Surveys were administered online during class time.

Ethical approval for the original study was obtained from the Hunter New England Health Human Research Ethics Committee (Ref. no. 09/11/18/4.01), The University of Newcastle Human Research Ethics Committee (Ref. no. H2010-0029), the Aboriginal Health and Medical Research Council (Ref. no. 776/11), the New South Wales Department of Education and Training State Education Research Approval Process (Ref. no. 2008 118), and the relevant Catholic Schools Offices. The trial is registered with the Australia and New Zealand Clinical Trials Register (Ref. no. ACTRN12611000606987).

The Australian Child Wellbeing Project (ACWP) is a cross-sectional survey of Australian school children. The ACWP is a nationally representative survey of children in years 4, 6 and 8 conducted in 2014. Data from children in Year 8 attending 180 government, catholic and independent schools across all states in Australia were used for this study. Surveys were administered online, with audio support for children with low literacy levels. Comprehensive details of the study methods are available elsewhere. $^{29}$

Ethical approval for the ACWP project was obtained from the Australian Council for Educational Research, Flinders University and the University of NSW. Further permissions were received from state/territorial authorities and dioceses and participation required written informed consent from parents and children. 


\section{Measures}

Back pain was measured slightly differently, but comparably in the two datasets. The questions were as follows:

- HSHF: 'In the last 6 months, how often have you had any pain in your back?' (never, rarely once or twice, about every month, about every week, more than $1 x /$ week, about every day)

- ACWP: 'In the last 6 months, how often have you had backache?' (rarely or never, about every month, about every week, more than $1 x /$ week, about every day)

Adverse health risk indicators were measured as per below, creation of dichotomous indicators was defined prior to analysis:

- Smoking:

o HSHF 'Have you smoked a cigarette in the last 4 weeks?’ (no, yes).

o ACWP 'On how many occasions have you smoked in the last 30 days?' dichotomized: never/1-2 times, 3-5, 6-9, 10-19, 20-39, 40 or more.

- Drinking:

o HSHF 'In the last 4 weeks, how many times have you had 5 or more alcoholic drinks in row?' dichotomized: none/once, twice, 3-6 times, 7 or more times.

o ACWP 'On how many occasions have you been drunk in the last 30 days?' dichotomized: never/1-2 times, 3-5, 6-9, 10-19, 20-39, 40 or more.

- Depression:

o HSHF 'How much of the time during have you felt down hearted or blue?' dichotomized: none/a little bit of the time, some of the time, a good bit of the time, most of the time, all of the time.

o ACWP 'In the past 6 months how often have you (had the following) feeling low?' dichotomized: rarely or never/about every month, about every week, more than $1 x /$ week, about every day.

- Anxiety:

o HSHF 'How much of the time during the past month have you been a very nervous person?' dichotomized: none/a little bit of the time, some of the time, a good bit of the time, most of the time, all of the time.

o ACWP 'In the past 6 months how often have you (had the following) feeling nervous?' dichotomized: rarely or never/about every month, about every week, more than $1 x /$ week, about every day.

- Missed school:

o ACWP 'Last term how many times have you missed school?' dichotomized: never, hardly ever/ $1 x /$ week, most days, every day.
We reported age, and selected sociodemographic measures to characterize the sample.

\section{Analysis}

Data were analysed using the Mantel-Haenszel test-fortrend using SPSS version 24. The analysis provides an indication of whether the odds of the adverse health indicators rise incrementally with increasing frequency of pain. Analyses were conducted separately in each dataset, and the findings compared to provide an indication of generalizability.

\section{Results}

\section{Sample characteristics}

Characteristics of the participants are presented in Table 1, both samples are similar with respect to age, gender proportion and language spoken at home. The fact that the HSHF study preferentially recruited schools from lower socioeconomic areas is reflected in the distribution of socioeconomic status variable, frequency of back pain was also higher in this dataset.

\section{Primary analysis}

In both the samples, the proportion of participants reporting smoking, drinking and missing school (ACWP data only) rose incrementally with increasing frequency of pain. The test-for-trend was significant for all risk factors in both samples. With respect to the mental health indicators, the proportion reporting feelings of depression or anxiety was much lower in the group that never or rarely experienced pain, but did not increase substantially in the other groups. Test-for-trend analyses for these mental health factors were statistically significant (Table 2 and Fig. 1). The absolute rates for all health risks were generally higher in the HSHF sample.

\section{Discussion}

\section{Main findings}

In both samples, more frequent back pain was associated with adverse health risk indicators. In most cases there was an apparent dose-response relationship as pain frequency increased, as indicated by the significant test-for-trend findings. These data indicate that back pain forms part of a wider picture of poor health risk profile. There were key differences between the datasets, in that the HSHF study preferentially recruited schools from socioeconomic strata lower than the Australian population norm, and that the mean age of the HSHF sample was approximately one year older. 
Table 1 Participant characteristics

\begin{tabular}{|c|c|c|}
\hline & $A C W P$ & HSHF \\
\hline Age range & $\begin{array}{l}14-15 \text { years } \\
n=3896\end{array}$ & $\begin{array}{l}14-16 \text { years } \\
n=2492\end{array}$ \\
\hline Female gender (\%) & $\begin{array}{l}48.8 \% \\
n=3896\end{array}$ & $\begin{array}{l}49.3 \% \\
n=2506\end{array}$ \\
\hline Socioeconomic status (\%) & $\begin{array}{l}18.0 \% \text { low } \\
36.1 \% \text { middle } \\
45.9 \% \text { high } \\
n=3896\end{array}$ & $\begin{array}{l}\text { Quintile } 1 ; 12.5 \% \\
\text { Quintile } 2 ; 30.9 \% \\
\text { Quintile } 3 ; 45.6 \% \\
\text { Quintile } 4 ; 10.5 \% \\
\text { Quintile } 5 ; 0.4 \% \\
n=2506\end{array}$ \\
\hline English spoken at home (\%) & $\begin{array}{l}92.9 \% \\
n=3893\end{array}$ & $\begin{array}{l}93.0 \% \\
n=2503\end{array}$ \\
\hline Quality of life* (mean (SD)) & $\begin{array}{l}7.62(1.69) \\
n=3881\end{array}$ & - \\
\hline Overall subjective health (\%) & $\begin{array}{l}34.8 \% \text { excellent } \\
54.9 \% \text { good } \\
9.0 \% \text { fair } \\
1.4 \% \text { poor } \\
n=3874\end{array}$ & - \\
\hline Back pain frequency & & \\
\hline Rarely or never & $51.9 \%$ & $66.7 \%$ \\
\hline Every month & $22.5 \%$ & $8.0 \%$ \\
\hline Every week & $11.1 \%$ & $8.7 \%$ \\
\hline$>1 x /$ week & $8.0 \%$ & $9.0 \%$ \\
\hline Every day & $\begin{array}{l}6.5 \% \\
n=3608\end{array}$ & $\begin{array}{l}7.7 \% \\
n=2075\end{array}$ \\
\hline
\end{tabular}

ACWP, Australian Child Wellbeing Project; HSHF, Healthy Schools Healthy Futures study; $n=$ total number of children who responded to the survey item. *Quality of life: $0=$ worst; $10=$ best.

Although the overall rate of adverse health risk indicators and frequency of back pain report was higher in this sample, the relationship with back pain frequency was the same. This provides support for generalizability of the findings.

There are several potential explanations for the relationships observed. These include causal pathways that flow in either direction, the action of confounders or complex feedback loops between pain and the other adverse health risk indicators. The cross-sectional structure of the data does not allow us to explore the form of these relationships. However, a narrow focus on aetiology may obscure important considerations. Report of frequent pain appears to be a marker of other potentially less visible issues, such as use of alcohol and tobacco, and poor mental health. This information could be used to identify an at-risk population to target interventions. For adolescents seeking care for back pain, these health risk factors may be related to poor long-term outcome of their back complaints. While we cannot be sure that prognostic relationships are causal, this points to a promising role for addressing these issues in the context of their clinical care. Conversely, it is plausible to suggest that the presence of pain is a barrier to the effectiveness of public health interventions aimed at promoting healthy lifestyle habits, related to physical activity, substance use and mental health. This may point to a place for addressing pain in the context of broader public health interventions.

\section{Interpretation}

These findings confirm previous literature that identifies associations between MSK pain and other indicators of poor health and health risk. As is the situation in research in adult populations, systematic reviews show a robust relationship between MSK pain and indicators of poor mental health, including depression and anxiety. ${ }^{11,25}$ There is inconsistency with regard to reported relationships between adolescent pain and alcohol use and tobacco. This study provides supporting evidence that such an association is real, a conclusion that is strengthened by the dose-response relationship between frequency and likelihood of drinking or smoking apparent in both datasets.

There are numerous potential explanations for the relationships between pain and health risk factors. Tobacco use may increase pain levels via physiological pro-inflammatory and vasoconstriction processes. ${ }^{30}$ Alternately experience of pain may see adolescents seeking analgesic effects of substance use as has been demonstrated in adult populations. ${ }^{31}$ Further, behavioural or cultural influences may lead to both pain report and alcohol use or smoking, e.g. a study has shown maternal influences on self-medication behaviours for pain management and coping in children. ${ }^{32}$ Similarly, anxiety or depression could be a consequence of frequent pain experience, or make pain more likely via behavioural or cognitive processes. Socioeconomic, or genetic factors may also provide a common explanatory pathway for psychological dysfunction and pain.

\section{Strengths and limitations}

The findings from this study come from two, relatively large and independently collected datasets. The fact that analyses reported similar relationships between the factors of interest is a strength of the study. Despite the combined sample of over 6000 participants, data was collected from only one country, which means that findings may not generalize beyond Australia. This may be of particular issue in countries where societal attitudes and access to alcohol and tobacco use are different. The study also makes use only of 
Table 2 Back pain frequency and health risks

\begin{tabular}{|c|c|c|c|c|c|}
\hline Back pain frequency & Smoking & Drinking & Depression & Anxiety & Missed school \\
\hline \multicolumn{6}{|l|}{ ACWP } \\
\hline Rarely or never & $1.7 \%$ & $2.5 \%$ & $39.7 \%$ & $56.2 \%$ & $9.3 \%$ \\
\hline Every month & $2.6 \%$ & $4.8 \%$ & $65.8 \%$ & $77.7 \%$ & $10.3 \%$ \\
\hline Every week & $4.0 \%$ & $4.3 \%$ & $75.1 \%$ & $81.5 \%$ & $12.0 \%$ \\
\hline$>1 \times /$ week & $4.9 \%$ & $7.0 \%$ & $80.1 \%$ & $82.6 \%$ & $19.8 \%$ \\
\hline Every day & $12.3 \%$ & $12.7 \%$ & $80.8 \%$ & $86.4 \%$ & $30.2 \%$ \\
\hline \multirow[t]{2}{*}{ a } & 67.7 & 51.9 & 349.7 & 196.4 & 75.0 \\
\hline & $P<0.01$ & $P<0.01$ & $P<0.01$ & $P<0.01$ & $P<0.01$ \\
\hline \multicolumn{6}{|l|}{ HSHF } \\
\hline Rarely or never & $9.3 \%$ & $12.6 \%$ & $78.1 \%$ & $86.5 \%$ & - \\
\hline Every month & $7.3 \%$ & $13.3 \%$ & $84.9 \%$ & $90.9 \%$ & - \\
\hline Every week & $10.0 \%$ & $17.2 \%$ & $85.6 \%$ & $92.8 \%$ & - \\
\hline$>1 x /$ week & $14.0 \%$ & $22.6 \%$ & $89.8 \%$ & $91.9 \%$ & - \\
\hline Every day & $20.8 \%$ & $21.3 \%$ & $90.0 \%$ & $89.4 \%$ & - \\
\hline \multirow[t]{2}{*}{ a } & 17.67 & 6.17 & 27.9 & 6.7 & - \\
\hline & $P<0.01$ & $P=0.01$ & $P<0.01$ & $P<0.01$ & b \\
\hline
\end{tabular}

ACWP, Australian Child Wellbeing Project; HSHF, Healthy Schools Healthy Futures project.

${ }^{\mathrm{a}}$ Mantel-Haenszel test-for-trend. ${ }^{\mathrm{b}}$ Missed school not available for HSHF.
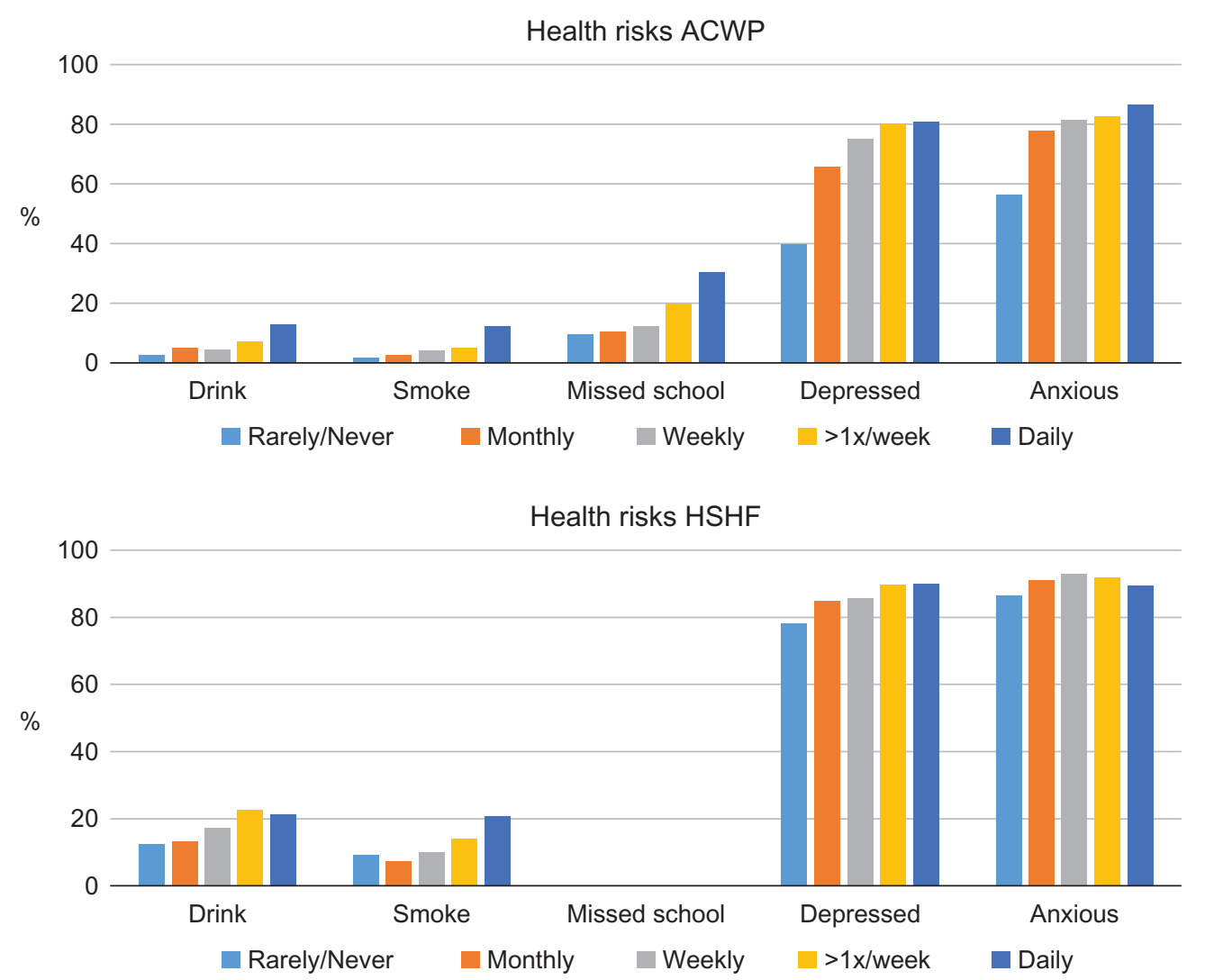

Fig. 1 Back pain frequency and health risks. ACWP, Australian Child Wellbeing Project; HSHF, Healthy Schools Healthy Futures project. *Missed school not available for HSHF. 
cross-sectional data, this means that the findings are unsuited to drawing conclusions about causal relationships.

\section{Implications}

These findings may have implications for clinical practice. They point to the fact that adolescents with frequent pain are at increased risk of other health problems. In the event that these other health risks influence the prognosis of painful conditions, then addressing health-related behaviours and mental health issues should form part of clinical management. Even if not causally related to the course of MSK pain, there is an argument that comprehensive health care of the individual should include management of these factors. In either case, screening for health-related risk behaviours and indicators of poor mental health is indicated in adolescents with frequent MSK pain.

From a research perspective this study suggests that consideration of behavioural health risks, and mental health should be incorporated into research aimed at understanding the pathology of MSK pain in adolescents. Understanding these relationships will also require longitudinal studies, with careful planning of the timing of data collection. These findings also point toward the potential for developing and evaluating treatment models that integrate best-practice clinical management of pain with interventions that target lifestyle, health-related behaviours and mental health. ${ }^{33}$

Finally, public health interventions commonly target substance use, and mental health in children and adolescents but they do not address pain. If it is the case that the same children that are at-risk of poor health outcomes due to substance use and/or mental health issues are also likely to experience pain more frequently, this may have implications for the design and content of public health interventions.

\section{Conclusions}

Adolescents that report more frequent pain are also more likely to report higher rates of adverse health risk behaviours and indicators of poor mental health. Back pain may play a role in characterizing poor overall health and risk of chronic disease in adulthood.

\section{References}

1 Levy S, Williams JF. Adolescent substance use: the role of the medical home. Adolesc Med State Art Rev 2014;25:1-14.

2 Home Office National Statistics (UK). Drug Misuse: Findings From the 2014/2015 Crime Survey for England and Wales. https://www.gov.uk/ government/uploads/system/uploads/attachment_data/file/ 450181/drug-misuse-1415.pdf2015.2015.
3 Taylor M, Collin SM, Munafò MR et al. Patterns of cannabis use during adolescence and their association with harmful substance use behaviour: findings from a UK birth cohort. I Epidemiol Community Health 2017;71:764-70.

4 Degenhardt L, Stockings E, Patton G et al. The increasing global health priority of substance use in young people. Lancet Psychiatry 2016;3:251-64.

5 Hall WD, Patton G, Stockings E et al. Why young people's substance use matters for global health. Lancet Psychiatry 2016;3:265-79.

6 Weissman DG, Schriber RA, Fassbender C. Earlier adolescent substance use onset predicts stronger connectivity between reward and cognitive control brain networks. Dev Cogn Neurosci 2015;16:121-29.

7 Institute for Health Metrics and Evaluation. GBD Compare I Viz Hub. https://vizhub.healthdata.org/gbd-compare/. University of Washington; 2016.

8 Copeland WE, Shanahan L, Costello EJ et al. Childhood and adolescent psychiatric disorders as predictors of young adult disorders. Arch Gen Psychiatry 2009;66:764-72.

9 Balogun O, Koyanagi A, Stickley A et al. Alcohol consumption and psychological distress in adolescents: a multi-country study. $J$ Adolesc Health 2014;54:228-34.

10 Kamper SJ, Henschke N, Hestbaek L et al. Musculoskeletal pain in children and adolescents. BrazJ Phys Ther 2016;20:275-84.

11 Kamper SJ, Yamato TP, Williams CM. The prevalence, risk factors, prognosis and treatment for back pain in children and adolescents: an overview of systematic reviews. Best Pract Res Clin Rheumatol 2016;30:1021-36.

12 Dissing KB, Hestbaek L, Hartvigsen J et al. Spinal pain in Danish school children-how often and how long? The CHAMPS StudyDK. BMC Musculoskelet Disord 2017;18:67.

13 Swain MS, Henschke N, Kamper SJ et al. An international survey of pain in adolescents. BMC Public Health 2014;14:447.

14 Henschke N, Harrison C, McKay D et al. Musculoskeletal conditions in children and adolescents managed in Australian primary care. BMC Musculoskelet Disord 2014;15:164.

15 Gobina I, Villberg J, Villerusa A et al. Self-reported recurrent pain and medicine use behaviours among 15-year olds: results from the international study. Eur J Pain 2015;19:77-84.

16 O'Sullivan PB, Beales DJ, Smith AJ et al. Low back pain in 17 year olds has substantial impact and represents an important public health disorder: a cross-sectional study. BMC Public Health 2012;12:100.

17 Brattberg G. Do pain problems in young school children persist into early adulthood? A 13-year follow-up. Eur J Pain 2004;8: 187-99.

18 Harreby M, Kjer J, Hesselsoe G et al. Epidemiological aspects and risk factors for low back pain in 38-year-old men and women: a 25year prospective cohort study of 640 school children. Eur Spine $J$ 1996;5(5):312-8.

19 Hestbaek L, Leboeuf-Yde C, Kyvik KO. Is comorbidity in adolescence a predictor for adult low back pain? A prospective study of a young population. BMC Musculoskelet Disord 2006;7:29.

20 Shiri R, Karppinen J, Leino-Arjas P et al. The association between smoking and low back pain: a meta-analysis. Am J Med 2010;87: e7-35. 
21 Heaps N, Davis MC, Smith AJ et al. Adolescent drug use, psychosocial functioning and spinal pain. J Health Psychol 2011;16:688-98.

22 Hestbaek L, Leboeuf-Yde C, Kyvik KO. Are lifestyle-factors in adolescence predictors for adult low back pain? A cross-sectional and prospective study of young twins. BMC Musculoskelet Disord 2006;7:27.

23 Kovacs FM, Gestoso M, Gil del Real MT et al. Risk factors for nonspecific low back pain in schoolchildren and their parents: a population based study. Pain 2003;103:259-68.

24 Law EF, Bromberg MH, Noel M et al. Alcohol and tobacco use in youth with and without chronic pain. J Pediatr Psychol 2015;40: 509-16.

25 Huguet A, Tougas ME, Hayden J et al. A systematic review with meta-analysis of childhood and adolescent risk and prognostic factors for musculoskeletal pain. Pain 2016;157:2640-56.

26 Caes L, Fisher E, Clinch J et al. The role of pain-related anxiety in adolescents' disability, and social impairment: ALSPAC data. Eur J Pain 2015;19:842-51.

27 Jussila L, Paananen M, Näyhä S et al. Psychosocial and lifestyle correlates of musculoskeletal pain patterns in adolescence: a 2-year follow-up study. Eur J Pain 2014;18:139-46.
28 Hodder RK, Freund M, Bowman J et al. Effectiveness of a pragmatic schoolbased universal resilience intervention in reducing tobacco, alcohol and illicit substance use in a population of adolescents: cluster-randomised controlled trial. BMJ Open 2017;7: e016060

29 Lietz P, O'Grady E, Tobin M et al. The Australian Child Wellbeing Project: Technical Report. Flinders University, the University of NSW and the Australian Council for Educational Research; 2015.

30 Ditre JW, Brandon TH, Zale EL et al. Pain, nicotine, and smoking: research findings and mechanistic considerations. Psychol Bull 2011; 137:1065-93.

31 Zale EL, Maisto SA, Ditre JW. Interrelations between pain and alcohol: an integrative review. Clin Psychol Rev 2015;37:57-71.

32 Jensen JF, Gottschau M, Siersma VD et al. Association of maternal self-medication and over-the-counter analgesics for children. Pediatrics 2014;133:e291-98.

33 Williams A, Wiggers J, O'Brien KM et al. A randomised controlled trial of a lifestyle behavioural intervention for patients with low back pain, who are overweight or obese: study protocol. BMC Musculoskelet Disord 2016;17:70. 